\title{
A Crystallographic Snapshot of SARS-CoV-2 Main Protease Maturation Process
}

\section{Gabriela D Noske ${ }^{1}$, Aline Minali Nakamura ${ }^{1}$, Victor O Gawriljuk ${ }^{1}$, Rafaela S Fernandes ${ }^{1}$, Gustavo M A Lima ${ }^{2}$, Higor V D Rosa ${ }^{1}$, Humberto D Pereira ${ }^{1}$, Ana C M Zeri ${ }^{3}$, Andrey F Z Nascimento ${ }^{3}$, Marjorie C L C Freire ${ }^{1}$, Glaucius Oliva ${ }^{1}$, Andre S Godoy ${ }^{1}$}

\author{
${ }^{1}$ Institute of Physics of Sao Carlos, University of Sao Paulo, Brazil; \\ ${ }^{2}$ BioMAX, MAX IV Laboratory, Lund, Sweden; \\ ${ }^{3}$ Brazilian Synchrotron Light Laboratory (LNLS), Campinas, Brazil; \\ oliva@ifsc.usp.br
}

SARS-CoV-2 is the causative agent of COVID-19. The dimeric form of the viral Mpro is responsible for the cleavage of the viral polyprotein in 11 sites, including its own $\mathrm{N}$ and $\mathrm{C}$ - terminus. The lack of structural information for intermediary forms of Mpro is a setback for the understanding of this process. Herein, we used X-ray crystallography to characterize an immature form of the main protease, which revealed major conformational changes in the positioning of domain-three over the active site, hampering the dimerization and diminishing its activity. We propose that this form preludes the cis-cleavage of $\mathrm{N}$-terminal residues. Using fragment screening, we probe new cavities in this form which can be used to guide therapeutic development. Furthermore, we characterized a serine site-directed mutant of the Mpro bound to its endogenous $\mathrm{N}$ and $\mathrm{C}$-terminal residues during the formation of the tetramer. We suggest this form is a transitional state during the $\mathrm{C}$-terminal trans-cleavage. This data sheds light in the structural modifications of the SARS-CoV-2 main protease during maturation, which can guide the development of new inhibitors.

Keywords: SARS-CoV-2, main protease, maturation process, inhibitors 